Enferm Bras 2021;20(1):20-37

doi: 10.33233/eb.v20i1.4232

\title{
ARTIGO ORIGINAL \\ O estresse intra-hospitalar e o aumento da pressão arterial entre acompanhantes de pacientes
}

Arimatéia Portela de Azevedo, M.Sc. ${ }^{*}$, Majorry de Oliveira Pereira ${ }^{* *}$, Adriana Sales de Abreu** ${ }^{* *}$ Daniel Guedes dos Santos ${ }^{* *}$, Michele Nascimento da Silva ${ }^{* *}$, Priscila Damasceno Frazão**, Rebeca Castro Moreira**, Wilzilene Coelho da Silva**

${ }^{*}$ Coordenadora da Comissão de Controle de Infecção $(\mathrm{CClH})$ da Fundação de Medicina Tropical Dr. Heitor Vieira Dourado, docente da Universidade Nilton Lins/UNINILTONLINS, Manaus, AM, ${ }^{\star \star}$ Enfermeiros assistenciais da Fundação de Medicina Tropical Dr. Heitor Vieira Dourado, Manaus, $A M,{ }^{* * *}$ Enfermeira especialista em Administração Hospitalar, Geriatria e Gerontologia e Saúde Pública e Docência em Enfermagem

Recebido em 26 de junho de 2020; aceito em 25 de fevereiro de 2021.

Correspondência: Arimatéia Portela de Azevedo, Rua São Judas Tadeu, 290/824 Bloco "C", Condominio Smile Parque das Flores 69028-360 Manaus AM

Arimatéia Portela de Azevedo: arimateia@fmt.am.gov.br Majorry de Oliveira Pereira: majorryoliveira7@gmail.com Adriana Sales de Abreu: adrianajg0227@gmail.com Daniel Guedes dos Santos: elguedes07@gmail.com Michele Nascimento da Silva: Michele.enf012@gmail.com Priscila Damasceno Frazão: prisciladamascenofrazo@gmail.com Rebeca Castro Moreira: rebeca.castro2712@gmail.com Wilzilene Coelho da Silva: wilzilenesilva.enfr@gmail.com

\section{Resumo}

Introdução: A internação gera alto grau de estresse e ansiedade na família. $O$ ambiente é percebido por eles como um espaço agressivo e ameaçador, pois 
evidencia o risco de morte do ente querido. Objetivo: Realizar acompanhamento de valores pressóricos de familiares cuidadores de pacientes internados para averiguar possíveis oscilações relacionadas ao estresse intra-hospitalar. Métodos: O presente estudo foi do tipo prospectivo, descritivo com abordagem quantitativa no qual foi realizado o registro pressórico dos acompanhantes de pacientes internados, no período de seis meses e averiguação dos fatores causais de maior estresse entre eles. Resultados: No período foram selecionados, por demanda espontânea, 48 acompanhantes de pacientes internados, dos quais $10,4 \%$ já declararam ser hipertensos e dos que declararam não ser hipertensos, $25 \%$ tiveram pico hipertensivo no primeiro dia como cuidador e 8,3\% no decorrer dos dias, 4,1\% teve elevação repentina da pressão arterial tendo que haver intervenção médica urgente. Conclusão: Os fatores estressores relacionados a permanência de um familiar cuidador em um ambiente hospitalar têm grande influência sobre o aumento dos valores pressóricos deles, principalmente ao se tratar de um hospital onde albergam pacientes com doenças infectocontagiosas.

Palavras-chave: acompanhante de paciente; infectologia; hipertensão arterial; enfermagem; familiar cuidador.

\section{Abstract \\ In-hospital stress and increased blood pressure among patients' companions}

Introduction: Hospitalization generates a high degree of stress and anxiety in the family. They perceive the environment as an aggressive and threatening space, as it highlights the risk of death of the loved ones. Objective: To monitor blood pressure values of family caregivers of hospitalized patients as an aid in interventions related to exposures to in-hospital stress. Methods: The present study was a prospective, descriptive study with a quantitative approach, which measured and recorded blood pressure of companions of hospitalized patients, within a period of six months, and investigated the main causes of high level of stress among them. Results: During the period, 48 companions of hospitalized patients were selected, due to spontaneous demand, $10.4 \%$ declared to be hypertensive and of those who declared not to be hypertensive, $25 \%$ had a hypertensive peak on the first day as a caregiver and $8.3 \%$ over the days, $4.1 \%$ 
had a sudden increase in blood pressure and urgent medical intervention was required. Conclusion: The stressors related to the stay of a family caregiver in a hospital environment have a great influence on the increase in blood pressure values, especially when dealing with a hospital where patients with infectious diseases are located.

Keywords: patient's companion; infectious diseases; high blood pressure; nursing; family caregiver.

\section{Resumen}

Estrés intrahospitalario y aumento de la presión arterial entre los acompañantes de los pacientes

Introducción: La hospitalización genera un alto grado de estrés y ansiedad en la familia. El entorno es percibido por ellos como un espacio agresivo y amenazante, ya que resalta el riesgo de muerte del ser querido. Objetivo: Monitorear los valores de presión arterial de cuidadores familiares de pacientes hospitalizados para averiguar posibles oscilaciones relacionadas al estrés hospitalario. Métodos: El presente estudio fue un estudio descriptivo prospectivo con un enfoque cuantitativo en el que se realizó el registro de presión arterial de los acompañantes de pacientes hospitalizados, dentro de un período de seis meses, y la determinación de los factores que desencadenan mayor estrés entre ellos. Resultados: Duurante el período, se seleccionaron 48 compañeros de pacientes hospitalizados, por demanda espontánea, el 10.4\% de los cuales declararon ser hipertensos y los que declararon no ser hipertensos, el $25 \%$ tuvieron un pico hipertensivo el primer día como cuidador y el $8.3 \%$ a lo largo de los dias, el 4,1\% tuvo un aumento repentino de la presión arterial y se requirió una intervención médica urgente. Conclusión: Los factores estresantes relacionados con la estadía de un cuidador familiar en un entorno hospitalario tienen una gran influencia en el aumento de los valores de la presión arterial, especialmente cuando se trata de un hospital donde se encuentran pacientes con enfermedades infecciosas.

Palabras-clave: acompañante del paciente; enfermedades infecciosas; presión arterial alta; enfermería; cuidador familiar. 
Introdução

A internação em um ambiente hospitalar gera alto grau de estresse e ansiedade na família. $\mathrm{O}$ ambiente é visto por eles como um local agressivo e ameaçador, pois evidencia o risco de morte do ente querido [1,2]. Tambem pode desencadear determinados comportamentos e sentimentos no familiar cuidador, como dúvidas, desamparo, desorganização mental, imobilização frente às decisões inesperadas e outras reações, como depressão ou doenças geradas pelo estresse e pela ansiedade $[2,3]$.

O estresse tem sido relacionado a sensações de tensão, ansiedade, medo e desconforto caracterizado por alterações psicofisiológicas que ocorrem quando o indivíduo é forçado a enfrentar situações que estão além de suas habilidades de enfrentamento $[4,5]$.

A tendência da interação nucleada na família, secundária a uma doença, é diretamente proporcional ao nível de incapacitação ou risco de morte. Nessa fase, a família passa por uma desestruturação, tendo que se ajustar a uma situação estranha ao seu cotidiano, que é conviver e aprender a lidar com o ambiente hospitalar e com os procedimentos terapêuticos relativos à doença $e$ estabelecer e manter bons relacionamentos com a equipe que presta cuidados $[6,7]$.

O processo de adoecimento e a hospitalização irão gerar um fato novo à família, fazendo com que ela reestruture sua rotina, além de submetê-la a um ambiente estranho para todos, pois a maioria das instituições hospitalares ainda não oferecem estrutura física nem acolhimento adequado [8].

Sabe-se que os cuidadores contribuem direta e indiretamente para o monitoramento, a manutenção e a gestão do autocuidado do paciente. Sendo assim, conhecer os fatores psicossociais (depressão, ansiedade e estresse) que podem estar presentes nos familiares e compreender o contexto em que 0 paciente e a família estão inseridos torna-se importante [9,10].

Estudos já realizados evidenciam que os familiares consideram como principais fatores estressores: a aparência do paciente internado, a necessidade de ventilação mecânica, a presença de curativos diversos, fios e aparelhos, bem como os ruídos dos equipamentos e da equipe. Além desses fatores, foram 
destacadas a gravidade do quadro clínico, a alteração do nível de consciência e a dificuldade e/ou ausência de comunicação [2,11].

A preocupação com o estresse se deve ao fato de ele afetar $90 \%$ da população mundial e estar fortemente relacionado às doenças do aparelho circulatório, que estão entre as primeiras causas de óbitos no Brasil. Ao estudarmos a natureza de um evento estressor, podemos defini-lo como quaisquer circunstâncias que ameaçam, ou são percebidas como ameaçadoras, ao bem-estar do indivíduo [12].

Os Descritores em Ciências da Saúde definem o estresse como o efeito desfavorável de fatores ambientais (estressores) sobre as funções fisiológicas de um organismo. O estresse fisiológico não resolvido e prolongado pode afetar a homeostase do organismo, levando a perdas ou afecções $[6,13]$.

Entre as diversas alterações provocadas pelo estresse, chama a atenção a hiper-reatividade do sistema nervoso simpático, denominada reatividade cardiovascular, definida como tendência a exibir a frequência cardíaca e PA exageradas $[6,14]$.

É comum encontrar os pacientes com muitos artefatos terapêuticos conectados a eles, dessa forma os pacientes conscientes e seus acompanhantes (familiares cuidadores) enfrentam, além do sofrimento físico, o psíquico por estarem em um ambiente estranho. Por sua vez, os familiares também sofrem e se assustam com o ambiente e com a gravidade do estado dos pacientes $[1,15,16]$.

Quando nos referimos à palavra estresse, deparamo-nos com alguns mitos de outrora, por exemplo, que só as pessoas ricas têm estresse, ou de que crianças não apresentam estresse. Porém, o organismo de um indivíduo desconhece classe econômica e também a idade. Assim, nos dias atuais, qualquer indivíduo pode desenvolver estresse. Desta forma, a equipe de enfermagem principalmente o enfermeiro tem grande responsabilidade em atender as necessidades que envolvam o paciente e seus familiares [17-19].

Do ponto de vista psicológico, o estresse pode ser definido como uma reação de um organismo, com componentes psicológicos e orgânicos, causada por alterações psicofisiológicas, quando o indivíduo faz frente a uma situação e tem sentimentos que incomodam, amedrontam, excitam, confundem ou mesmo o tornam feliz $[14,20,21]$. 
Os sentimentos vivenciados pelo familiar acompanhante como o medo, a ansiedade e a insegurança são gerados, muitas vezes, pela falta de apoio, de atenção e de informações da equipe de saúde [4,22]. As adversidades interferem no equilíbrio familiar afetando a dinâmica de todos os seus membros [21,23].

Um conceito importante e que pode ser fator gerador de estresse em cuidadores é a sobrecarga, por se tratar de pacientes com acompanhantes fixos e sem auxílio de outros membros da família [17,24].

A hospitalização geralmente é tida como desagradável tanto para o paciente quanto para o familiar, pois é necessário que ele abandone seu lar, seu trabalho e a família para poder cuidar do familiar internado [25-27].

A doença e a incapacidade são experiências comuns às famílias e representam um grande desafio, uma vez que os problemas psicossociais ocasionados por uma pessoa com dependência desencadeiam impacto sobre todo o sistema familiar [21,15]. A doença provoca um impacto e desestrutura o universo familiar. O sofrimento em ver um ente querido ameaçado e sujeito a tratamentos agressivos, dor, dependência, provoca uma série de sentimentos controversos $[4,15]$.

Independente das intervenções realizadas pelos familiares acompanhantes diante do processo de cuidados no ambiente hospitalar, eles experimentam sensações positivas e negativas. Os aspectos negativos estão relacionados às alterações que ocorrem na sua vida diária, a interferência na sua rotina, ao cansaço físico, emocional e problemas financeiros. Por outro lado, os cuidadores sentem satisfação por poder ajudar seus familiares [21,22].

Estudos apontam que esses cuidadores possuem mais chances de apresentarem sintomas psiquiátricos e problemas de saúde, entre os quais hipertensão arterial, desordens digestivas e respiratórias, depressão, além de vivenciarem conflitos familiares e problemas no trabalho com maior frequência, em comparação a pessoas da mesma idade que não exercem tal função [14,25].

O estresse, por estimular o sistema nervoso simpático, afeta também a pressão arterial, fazendo com que haja um aumento da frequência cardíaca e da força contrátil dos batimentos cardíacos, assim como da resistência periférica, aumentando o risco de Doença Arterial Coronariana (DAC) [22]. A manutenção crônica de altos níveis de estresse pode estar associada ao desenvolvimento de 
doenças, como insuficiência cardíaca, aterosclerose, isquemia e hipertensão arterial (HA) [25,26].

É importante frisar que o estresse afeta tanto uma pessoa que não possui doenças do aparelho circulatório, tanto a que possui. A hipertensão arterial tem sido associada com uma aumentada reatividade vascular durante e após períodos agudos de estresse induzido [25,27]. Evidenciou-se também aumentos no débito cardíaco, na vasoconstricção, na resistência vascular periférica, na atividade nervosa simpática e, por conseguinte, elevações anormais na pressão arterial em humanos que se encontravam em condições de estresse [25].

Os familiares acompanhantes estão mais propensos a adoecer do que os outros familiares e amigos, devido a permanência no ambiente hospitalar, a negação do cuidado de si e o contato direto com a pessoa dependente de cuidados [21]. As dificuldades encontradas pelos acompanhantes são desencadeadas, na sua grande maioria, pela falta de esclarecimentos da situação clínica que envolve o processo saúde-doença por parte dos profissionais $[9,19]$.

Sendo assim, é importante que os profissionais da enfermagem, sobretudo o enfermeiro, invistam em ações de cuidado demonstrando interesse, consideração e sensibilidade em relação ao doente e seu familiar [13,20]. O interesse em participar do cuidado e a inter-relação do acompanhante com a equipe de enfermagem são elementos facilitadores para o processo de hospitalização aliado ao bem-estar do doente e seus familiares [3,18,4]. A participação mútua da equipe de saúde e do acompanhante fortalece o elo da tríade, ou seja, profissional/acompanhante/paciente para amenizar a angústia e a tristeza de estar nesta situação [16]. A interação e apoio da equipe com a família gera bem-estar e conforto, portanto, pode minimizar esse momento difícil e desgostoso para a mesma [23]. A enfermagem busca promover uma interação com o acompanhante dando-Ihe um suporte emocional e cognitivo, com orientações e informações sobre as condições da doença e trabalhando uma melhor percepção no cuidado [23,24]. Criar estratégias para o enfrentamento dos desafios relacionados com o processo de hospitalização pode ser uma maneira de a equipe de enfermagem auxiliar o familiar acompanhante neste processo [4]. 


\section{Objetivo}

Registrar valores pressóricos de acompanhantes de pacientes internados para averiguar possiveis oscilações relacionadas ao estresse intra-hospitalar Métodos

O presente estudo foi do tipo prospectivo, descritivo com abordagem quantitativo, no qual foi possível realizar o registro pressóricos dos acompanhantes de pacientes internados em um hospital referência em infectologia de Manaus e averiguar os fatores causais de maior estresse entre acompanhantes tais como: alimentação, diagnóstico do paciente, medo de se contaminar com alguma doença infecciosa durante os cuidados, limpeza do ambiente, cheiro do hospital. E fatores externos: familiares, financeiros, entre outros.

A pesquisa foi realizada com uma amostra de 48 acompanhantes de pacientes, acima de dezoito anos de idade, que estavam internados no período proposto pela pesquisa, em qualquer enfermaria, e que preenchessem o Termo de Consentimento Livre e Esclarecido (TCLE). Não foram incluídos no estudo acompanhantes que permaneceram menos de cinco dias junto ao paciente por não haver, nesses casos, possibilidade de se fazer uma curva de tendência da pressão arterial.

Para que houvesse fidelidade nas respostas, cada acompanhante teve a mensuração da pressão arterial realizada duas vezes ao dia, no mesmo horário, nos dois braços, utilizando-se do mesmo aparelho e sendo realizada pelo mesmo profissional. Foi aferida a pressão arterial dos acompanhantes por sete dias consecutivos, desde o primeiro dia em que adentrou no hospital. Tais registros permitiram a averiguação dos possíveis fatores causais de maior estresse entre esses acompanhantes.

As informações utilizadas neste estudo foram coletadas diretamente do participante do estudo após assinatura do TCLE. Após aceitação em participar, 
foi aferida a pressão arterial e realizado o preenchimento de um questionario contendo perguntas com respostas fechadas: informações sociodemograficas, sobre as ansiedades e preocupações do mesmo trazidas para o hospital ou adquiridas no ambiente hospitalar, história de doenças crônico degenerativas (principalmente hipertensão).

Para maior proteção do participante da pesquisa, o questionário teve como identificador apenas um número sequencial. Após levantamento dos dados obtidos, foi realizado levantamento estatístico e discussão de todo o material. A apresentação dos resultados se fez de forma descritiva, quantitativa. Para o tratamento dos dados foi utilizado o software SPSS versão 19.

A pesquisa teve início após a aprovação pelo Comitê de Ética em Pesquisa (CEP), sob o CAAE número 16525519.7.0000.0005, Número do Parecer: 3.512.250, de acordo com a resolução 466/12 do Ministério da Saúde/CEP

\section{Resultados e discussão}

Foram selecionados, por demanda espontânea, 48 acompanhantes de pacientes internados, dos quais $12,5 \%$ já se declararam ser hipertensos e dos que declararam não ser hipertensos, $25 \%$ tiveram pico hipertensivo no primeiro dia de internação e 31,3\% no decorrer da internação.

Tabela I - Perfil sociodemográfico dos acompanhantes de pacientes internados durante o período proposto pela pesquisa

\begin{tabular}{lll}
\hline Perfil dos acompanhantes & $\left(\mathbf{n}^{\circ}\right)$ & $(\mathbf{\%})$ \\
\hline Homens & 12 & 25,0 \\
Mulheres & 36 & 75,0 \\
Idade entre 20 a 50 anos & 36 & 75,0 \\
Ensino médio completo & 27 & 56,3 \\
Acompanhante vindos de outra didade & 21 & 43,7 \\
Acompanhante fixo do paciente & 20 & 41,7 \\
Acompanhante hipertenso & 6 & 12,5 \\
\hline
\end{tabular}

Fonte: dados da pesquisa

Neste estudo percebeu-se que os resultados coadunam com o mesmo informado pelo autor supracitado [21], pois alguns acompanhantes não tinham nenhum apoio da família e acabavam se tornando o único acompanhante do 
paciente (acompanhante fixo), não tendo a oportunidade de descanso fora do ambiente hospitalar, causando sobrecarga sobre o mesmo (Tabela I).

Investigando essa mesma temática fizeram outro estudo com acompanhantes de pacientes internados em uma unidade de clínica médica de um hospital público e de ensino no Rio Grande do Sul onde constataram que a hospitalização prolongada geralmente tem como consequência a sobrecarga de um familiar cuidador. Por diversos fatores, a responsabilidade do cuidado, recai por vezes, em um único membro da família [4]. Muitos familiares não contam com o apoio dos demais membros da família para acompanhar o paciente no hospital, realizando estas atividades sozinhos [3].

Estudo [18] realizado com familiar cuidador de pacientes internados em um hospital referência em infectologia mostrou que a maioria (74\%) moravam na própia cidade onde o paciente fora internado, mas, $20 \%$ moravam em outras cidades dentro do mesmo estado e 6\% eram oriundos de outros estados brasileiros. Morar fora do domicílio onde o paciente foi internado pode ser um fator estressor em virtude da impossibilidade de troca quando o estado físico e emocional ficarem esgotados. Tal situação pode ser um fator desencadeantes de estresse.

Em outro estudo realizado com acompanhantes de pacientes internados no Hospital Estadual de Ribeirão Preto/SP, verificou-se que os participantes são em geral mulheres de meia idade. Poucos homens participaram do grupo, o que remete a pensar no cuidado como uma tarefa historicamente construída para ser feminina [9].

Novamente, neste estudo, os resultados de Dahadaha et al. [9] são similares, pois a maioria dos acompanhantes eram do sexo feminino, grande parte dessa porcentagem foi composta por mães e irmãs do paciente (Tabela I). 


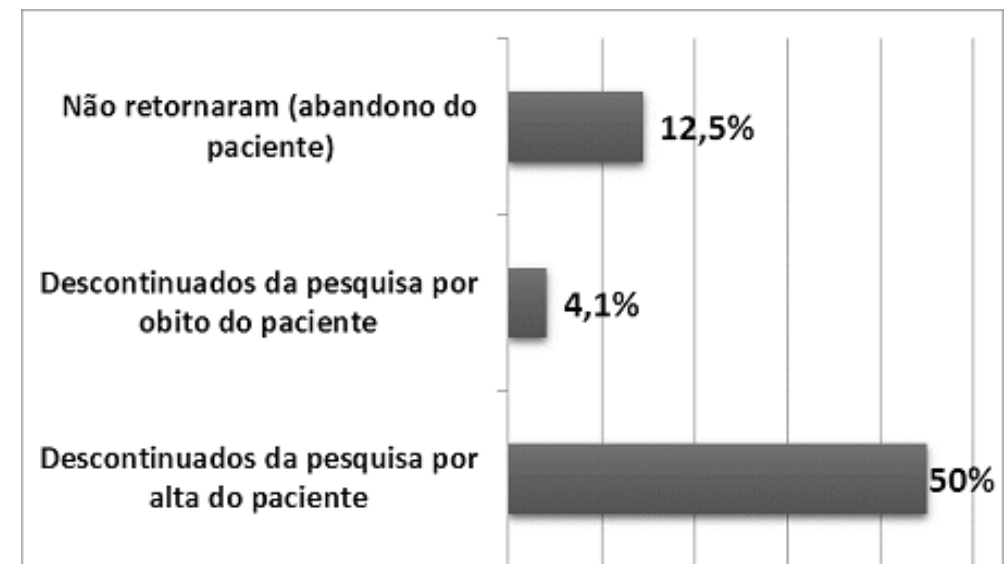

Fonte: dados da pesquisa

Gráfico 1 - Relatos dos motivos da descontinuidade, como acompanhantes, de alguns familiares cuidadores

Tabela II - Possíveis fatores de risco intra-hospitalar ou familiar relacionados ao aparecimento de aumento nos valores pressóricos entre os investigados no primeiro dia de internação

\begin{tabular}{llll}
\hline $\begin{array}{l}\text { Fonte do } \\
\text { problema }\end{array}$ & Motivos relatados & N & $\%$ \\
\hline De ordem & 1- Preocupação com o paciente & 16 & 33,3 \\
hospitalar & 2- Sofrimento ao ver a piora do quadro do paciente & 9 & 18,8 \\
& 3- Mal-estar, medo de sangue & $\mathbf{8}$ & $\mathbf{1 6 , 7}$ \\
& 4- Falta de poltrona & 6 & 12,5 \\
& 5- Falta de informacões dos profissionais & 6 & 12,5 \\
\hline De ordem & 1- Dificuldades/desentendimento com o paciente & 23 & 47,9 \\
familiar & 2- Preocupações com a familia & 22 & 45,8 \\
& 3- Instabilidade financeira & 4 & 8,3 \\
\hline
\end{tabular}

Fonte: dados da pesquisa

Com base nas informações obtidas através do questionário foi possível pontuar alguns dos possíveis fatores que influenciaram nos aumentos dos valores pressóricos de cada acompanhante logo no início da internação. Este estudo evidenciou tanto fatores intra-hospitalares como os fatores externos, que por vezes incluíam situações que não tinham nada a ver com o paciente, e sim com os problemas do cotidiano do acompanhante, uma vez que para se tornar acompanhante um indivíduo acaba deixando de lado seus próprios interesses e responsabilidades (Tabela II).

Houve acompanhantes que relataram fatores estressores tanto no ambiente hospitalar quanto fatores externos. O aumento da pressão arterial de outro grupo de acompanhantes já tinha como base somente fatores intrahospitalares (Tabela III). 
Alguns autores defendem a relação do aumento da reatividade cardiovascular com a expressão de raiva para fora, outros concluem que tal aumento está relacionado com a expressão da raiva para dentro [23,24].

Tabela III - Possíveis fatores de risco intra-hospitalar e familiar relacionados ao aparecimento de aumento nos valores pressóricos no decorrer da internação

\begin{tabular}{llll}
\hline $\begin{array}{l}\text { Fonte do } \\
\text { problema }\end{array}$ & Motivos relatados & $\mathbf{( n}^{\circ} \mathbf{)}$ & $\mathbf{( \% )}$ \\
\hline De ordem & 1- Desconforto na hora de dormir & 20 & 41,7 \\
hospitalar & 2- Dores de cabeça e no corpo devido a poltrona & 17 & 35,4 \\
& 3- Estresse com r otina e com alquns profissionais & 8 & 16,7 \\
\hline De ordem & 1- Desentendimento com o paciente & 26 & 54,1 \\
familiar & 2-Medo de se contaminar durante os cuidados & 7 & 14,6 \\
& 3- Instabilidade financeira & 7 & 14,6 \\
& 4-Preocupações com a família & 6 & 12,5 \\
& 5- Desemprego dos filhos & 4 & 8,3 \\
& 6-Término de relacionamento & 1 & 2,1 \\
\hline
\end{tabular}

Fonte: dados da pesquisa

Foi observado ao longo da coleta de dados que os acompanhantes tiveram muitas dificuldades em se adaptar ao ambiente hospitalar. Relataram que além de terem que aprender a lidar com o paciente, ainda tiveram que conviver com o desconforto, cansaço, maus tratos pela parte dos profissionais e problemas de saúde já instalados. Os fatores de estresse relatados pelos participantes deste estudo no decorrer da internação são, provavelmente, continuidade dos fatores estressores do início da internação (Tabela III).

Além de todos esses fatores, esses familiares cuidadores informaram que trouxeram uma carga de preocupações com fatores externos tais como: familiar, trabalho, estudo e que tais angústias sempre estavam presentes, potencializando ainda mais o aparecimento de doenças ou pioras de doenças já instaladas em seus organismos (Tabela III).

Outro autor concluiu que uma das formas de compreender os fatores emocionais associados à hipertensão arterial, como é o caso do stress e da raiva, é o entendimento do conceito de reatividade cardiovascular, que se refere às modificações de pressão arterial ou de frequência cardíaca, decorrentes de estímulos específicos. Embora a tendência geral da maioria das pessoas seja de elevações de pressão arterial frente a situações de stress, indivíduos com hipertensão apresentam elevações maiores e mais frequentes do que pessoas sem este diagnóstico em situações similares [11,28]. 


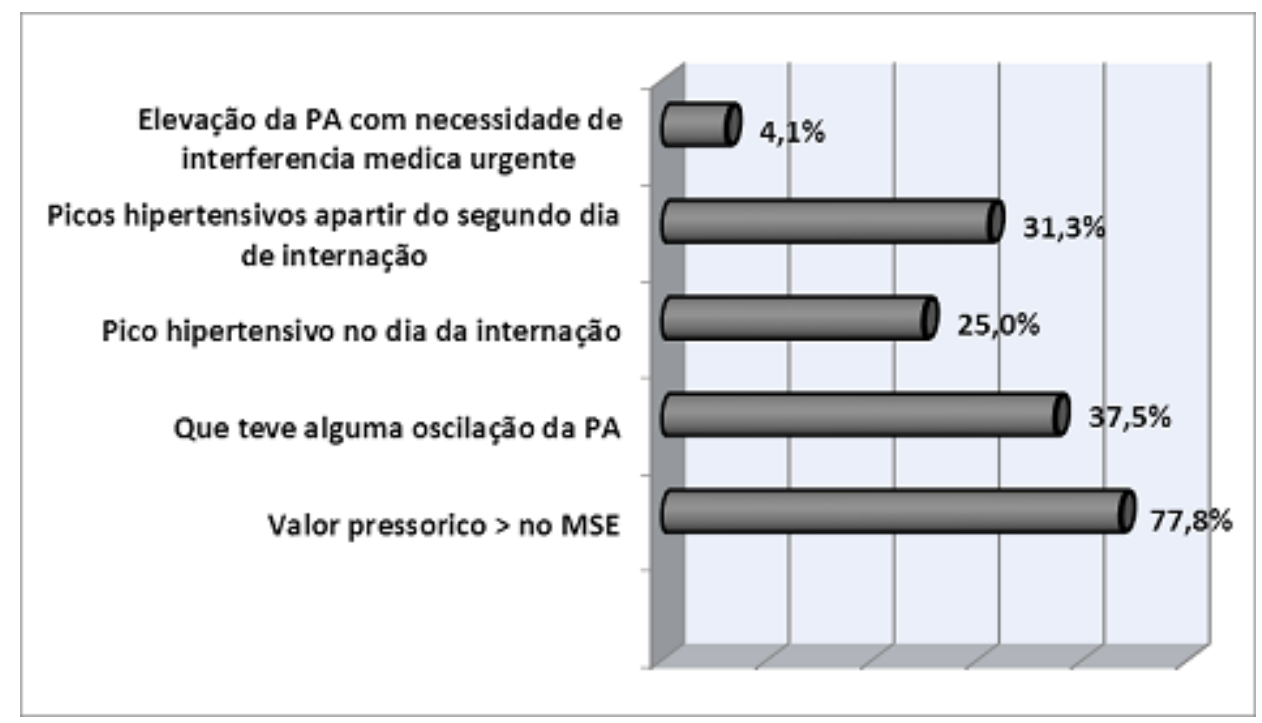

Fonte: Dados da pesquisa

Gráfico 2 - Registro das intercorrências de oscilação dos valores pressóricos ocorridos entre familiares cuidadores durante a estada no ambiente hospitalar

Conforme a monitorização dos valores pressóricos dos acompanhantes (familiares cuidadores) de pacientes internados foi possível detectar que a pressão arterial deles oscilou em diversos momentos durante a estadia do paciente no ambiente hospitalar, mas foi percebido que houve maior elevação nos primeiros dias de internação do paciente. Alguns dos participantes que tiveram uma elevação alarmante da pressão arterial (PA) foram encaminhados ao pronto atendimento do hospital onde ocorreu a pesquisa (Gráfico 2).

Outro fator importante a ser destacado é que no momento em que acontecia uma piora no quadro do paciente os acompanhantes passaram por instabilidade emocional com base em preocupação e aflição, fatores estes que fizeram com que a pressão arterial dos mesmos se elevasse.

Ao lado de outros fatores como hereditariedade, vida sedentária e nutrição inadequada, stress e raiva estão relacionados à hipertensão arterial. O stress pode ser compreendido como uma resposta do organismo da qual fazem parte fatores físicos, psicológicos, mentais e hormonais originada pela necessidade de manejo de qualquer situação, positiva ou negativa, que naquele momento representa ameaça [24,29]. 
Sabe-se que nas últimas décadas tem-se valorizado o papel do familiar cuidador como facilitador no restabelecimento da saúde de pacientes internados em unidades hospitalares, assim como agente acelerador no processo de reabilitação. Mediante o que foi pesquisado por este estudo, chegamos as seguintes conclusões:

No período de internação, o familiar cuidador passa a se dedicar ao paciente, esquecendo muita das vezes de suas próprias necessidades ou limitações. Nesse cenário que ele se insere, está sujeito a se deparar com qualquer tipo de situação relacionada a um ambiente hospitalar, onde nem sempre esse acompanhante terá preparo físico ou psicológico.

Durante este estudo foi possível perceber que, além dos fatores causais do estresse intra-hospitalar, a condição clinicopatológica do paciente foi um grande agravante na instabilidade pressórica desses acompanhantes. Pois quando havia piora do quadro clínico do paciente, era bem perceptivo a preocupação e pensamentos negativos tomarem conta dos mesmos. Essas condições trouxeram desequilíbrio no bem-estar prejudicando seu sono e repouso, suas condições psicológicas e fisiológicas, pressão arterial, e até mesmo prejudicando outras doenças já instaladas.

Muitos participantes deste estudo não tinham nenhuma condição psicológica ou física de estar como acompanhantes. Mas, eram circunstancialmente os que podiam ficar como cuidador. Diversos fatores tornaram essa situação obrigatória, como: a falta de familiares na cidade (pois essas pessoas vieram do interior ou outras cidades), a falta de apoio da família, a grande preocupação que acabava impossibilitando esse acompanhante de sair de perto do paciente, a falta de recursos financeiros, entre outras situações.

Também foram feitos registros de relatos dos mesmos e algumas perguntas relacionadas a possíveis fatores causais do estresse. Percebeu-se que muitos precisavam externar as situações estressoras em que se encontravam. 
Os familiares cuidadores são, além de muito importantes na recuperação dos pacientes, são seres humanos e devem ser tratados de forma humanizada ao estarem em uma unidade hospitalar.

Portanto, após o levantamento de todos os dados, chegamos à conclusão que possivelmente os fatores estressores relacionados a permanência em um ambiente hospitalar podem ter grande influência sobre o aumento dos valores pressóricos de um indivíduo, principalmente ao se tratar de um hospital onde alberga pacientes com doenças infectocontagiosas, com $80 \%$ de pacientes que convivem com o vírus do HIV.

\section{Agradecimentos}

Agradecemos a Fundação de Medicina Tropical Dr. Heiror Vieira Dourado pela oportunidade dada para que este estudo fosse possível.

\section{Financiamentos}

Este estudo foi custeado com recursos dos próprios autores

\section{Referências}

1. Arantes RKM, Salvagioni DAJ, Araujo JP, Roecker S. Educação que produz saúde: atuação da enfermagem em grupos de hipertensos. Rev Enferm UFSM 2015;5(2):21323. https://periodicos.ufsm.br/reufsm/article/view/13472/pdf

2. Barth AA, Weigel BD, Dummer CD, Machado KC, Tisott TM. Estressores em familiares de pacientes internados na unidade de terapia intensiva. Rev Bras Ter Intensiva. 2016;28(3):323-329. Disponível em: http://www.scielo.br/pdf/rbti/v28n3/0103-507X-rbti28-03-0323.pdf

3. Azevedo AP, Cristino JS, Viana MF, Medeiros FP, Azevedo LS. Educação em saúde para acompanhantes de pacientes internados. Rev Enferm UFPE 2018,12(4):1168-73. https://doi.org/10.5205/1981-8963-v12i4a230649p1168-1173-2018

4. Beuter M, Brondami CM, Szareski C, Cordeiro FR, Roso CC. Sentimentos de familiares acompanhantes de adultos face ao processo de hospitalização. Esc Anna Nery 2012;16(1):134-40. https://doi.org/10.1590/S1414-81452012000100018

5. Costa JB, Felicetti CR, Costa CRLM, Miglioranza DC, Osaku EF, Versa GLGS, et al. Fatores estressantes para familiares de pacientes criticamente enfermos de uma 
unidade de terapia intensiva. J Bras Psiquiatr 2010;59(3):182-9. Disponível em: http://www.scielo.br/pdf/ibpsiq/v59n3/a03v59n3

6. Cachos JRE, Santos TV, Gon RS. Resposta da pressão arterial ao estresse agudo traumático em urgências ortopédicas. Rev Bras Clin Med 2013;11(1):17-20. http://files.bvs.br/upload/S/1679-1010/2013/v11n1/a3382.pdf

7. Montefusco SAR, Bachion MM. Manutenção do lar prejudicada: diagnóstico de enfermagem em familiares de pacientes hospitalizados com doenças crônicas. Rev Eletr Enf 2011;13(2):182-9.

https://repositorio.bc.ufg.br/xmlui/bitstream/handle/ri/1264/14201-63419-1PB.pdf? Sequence $=1$ \&isAllowed $=y$

8. Vieira GB, Alvarez AM, Girondi JBR. O estresse do familiar acompanhante de idosos dependentes no processo de hospitalização. Rev Eletr Enf 2011;13(1):78-89. https://www.revistas.ufg.br/fen/article/ view/8719/9163

9. Dahdah DF, Carvalho AMP, Delsimc JC, Gomesd BR, Migueld VS. Grupo de familiares acompanhantes de pacientes hospitalizados: estratégia de intervenção da Terapia Ocupacional em um hospital geral. Cad Ter Ocup 2013;21(2):399-404. https://doi.org/10.4322/cto.2013.041

10. Lacerda MS, Cirelli MA, Bottura ALLB, Lopes JL. Ansiedade, estresse e depressão de familiares de pacientes com insuficiência cardíaca. Rev Esc Enferm USP 2017;51:e03211. https://doi.org/10.1590/s1980-220x2016018903211

11. Duarte OO, Faria WRC, Pinto FM, Nakaoka VYES, Kashiwabara TGB. Tratamento ambulatorial da hipertensão arterial sistêmica - revisão de literatura. Revista UNINGÁ 2014;1(2):22-9. http://revista.uninga.br/index.php/uningareviews/article/view/1492/1107

12. Gomes CM, Capelarri C, Pereira DSG, Volkart PR, Moraes AP, Jardim V, Bertuo M. Estresse e risco cardiovascular: intervenção multiprofissional de educação em saúde. Rev Bras Enferm 2016;69(2):351-9. https://doi.org/10.1590/0034-7167.2016690219i

13. Guerin PC, Grasland F, Marion LT, Bénédicte G. Diffusion of foreign eurocoins in France, 2002-2012. Population et sociétés. INED ; 2012. https://hal.archivesouvertes.fr/hal-01651881/document

14. Junior EL, Neto EL. Hipertensão arterial: aspectos comportamentais - Estresse e migração. Rev Bras Hipertens 2010;17(4):210-25. http://departamentos.cardiol.br/dha/revista/17-4/revisao-hipertensao.pdf

15. Zattar LC, Boing AF, Gieh MWC, d'Orsi E. Prevalência e fatores associados à pressão arterial elevada, seu conhecimento e tratamento em idosos no sul do Brasil. Cad Saúde Pública 2013;29(3):507-21. https://doi.org/10.1590/S0102-311X2013000300009

16. Lima SF, Vitor ACS, Moraes MS, Pereira F. Acompanhantes de pacientes hospitalizados e suas demandas no cuidado. Rev Cien Escol Estad Saud Publ Cândido Santiago-RESAP 2015;1(1):52:52-62. Disponível em: http://www.revista.esap.go.gov.br/index.php/resap /article/download/13/21 
17. Maronesi LC, Silva NR, Cantu SO, Santos AR. Indicadores de estresse e sobrecarga em cuidadores formais e informais de pacientes oncológicos. Estud Pesqui Psicol 2014;14(3):877-92. Disponível em: https://www.redalyc.org/pdf/4518/451844509010.pdf

18. Azevedo AP, Sena RPS, Diniz ACS, Conceição AC, Azevedo AM, Araújo KM et al. Recursos físicos e insumos disponíveis como medidas de controle intra-hospitalar aplicadas para a prevenção da transmissão do mycobacterium tuberculosis. Braz J Health Rev 2020;3(2):2168-81.

19. Mendes CRS, Miranda MC, Lima FET, Brito EAWS, Freitas I, Matias EO. Prática de autocuidado de pacientes com hipertensão arterial na atenção primária de saúde. Rev Rene 2016;17(1):52-9. https://www.redalyc.org/pdf/3240/324044160008.pdf

20. Prado CEP. Estresse ocupacional: causas e consequências. Rev Bras Med Trab 2016;14(3):275-84. Disponível em: http://www.anamt.org.br/site/upload arquivos/revista brasileira de medicina do trabalho volume 14 n\%C2\%BA 31312 20161657237055475.pdf\#page $=107$

21. Passos SSS, Pereira A, Nitschke RG. Cotidiano do familiar acompanhante durante a hospitalização de um membro da família. Acta Paul Enferm 2015;28(6):539-45. https://doi.org/10.1590/1982-0194201500090

22. Quintana JF. A relação entre hipertensão com outros fatores de risco para doenças cardiovasculares e tratamento pela psicoterapia cognitivo comportamental. Rev SBPH 2011;14(1). http://pepsic.bvsalud.org/scielo.php?script=sci arttext\&pid=S151608582011000100002

23. Richter S, Deter HC, Rudat M, Schächinger H, Zimmermann-Viehoff F, Weber C. Anger and cardiovascular startle reactivity in normotensive young males. Int J Psychophysiol 2011;79(3):364-70. https://doi.org/10.1016/j.ijpsycho.2010.12.004

24. Sanz J, García-Vera MP, Espinosa R, Fortún M, Magán I, Segura J. Psychological factors associated with poor hypertension control: differences in personality and stress between patients with controlled and uncontrolled hypertension. Psychol Rep 2010;107(3):923-38. https://doi.org/10.2466/09.15.20.PR0.107.6.923-938

25. Silva MFL, Campbell CSG, Brito AF, Silva AS, Santos MAP, Formiga MNR, Moreira SR. O volume de exercícios resistidos influencia a reatividade da pressão arterial ao estresse. Rev Bras Med Esporte 2015;21(6):438-41. https://doi.org/10.1590/1517869220152106118724

26. Vitória AL, Assis CL. Vivências e estratégias de enfrentamento em acompanhantes de familiar hospitalizado em uma unidade hospitalar do município de Cacoal-RO. Aletheia 2015;46:16-33. https://www.redalyc.org/pdf/1150/115048330003.pdf

27. Silva SSBE, Oliveira SFSB, Oierin AMG. O controle da hipertensão arterial em mulheres e homens: uma análise comparativa. Rev Esc Enferm USP 2016;50(1):50-8. Disponível em: http://www.periodicos.usp.br/reeusp /article/view/112689/110605

28. González-Fraile E, Ballesteros J, Rueda J-R, Santos-Zorrozúa B, Solà I, McCleery J. Remotely delivered information, training and support for informal caregivers of people 
with dementia. Cochrane Database of Systematic Reviews 2021;(1):CD006440.

https://doi.org/10.1002/14651858.CD006440.pub3

29. Hui D, Hannon BL, Zimmermann C, Bruera E. Improve the results of the patient and caregiver in oncology: palliative care directed by the team, timely and targeted. CA Cancer J Clin 2018;68:356-76.

https://acsjournals.onlinelibrary.wiley.com/doi/full/10.3322/caac.21490 